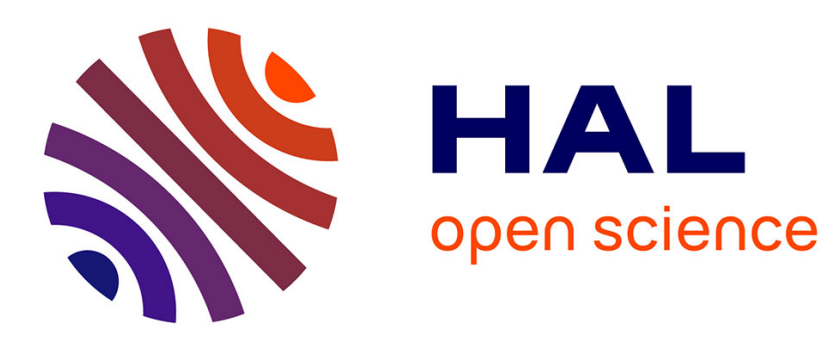

\title{
Extreme Value Theory and Copulas: Reinsurance in the Presence of Dependent Risks
}

\author{
Queensley C Chukwudum
}

\section{To cite this version:}

Queensley C Chukwudum. Extreme Value Theory and Copulas: Reinsurance in the Presence of Dependent Risks. 2018. hal-01855971

\section{HAL Id: hal-01855971 \\ https://hal.science/hal-01855971}

Preprint submitted on 9 Aug 2018

HAL is a multi-disciplinary open access archive for the deposit and dissemination of scientific research documents, whether they are published or not. The documents may come from teaching and research institutions in France or abroad, or from public or private research centers.
L'archive ouverte pluridisciplinaire HAL, est destinée au dépôt et à la diffusion de documents scientifiques de niveau recherche, publiés ou non, émanant des établissements d'enseignement et de recherche français ou étrangers, des laboratoires publics ou privés. 


\title{
Extreme Value Theory and Copulas: Reinsurance in the Presence of Dependent Risks
}

\author{
Queensley C. Chukwudum ${ }^{1}$ \\ Pan African University of Basic Sciences, Technology and Innovation \\ queensleyv@yahoo.com
}

August, 2018

\begin{abstract}
An insurer's ability to accurately estimate the accumulation of risk, particularly in the right hand tail, is vital in ensuring that his risk appetites matches his risk exposures. This paper, therefore, focuses on the modeling of the extremal dependence structure between insurance risks using the Generalized Pareto distribution and the copula technique. The results obtained after comparing the dependence between large losses from two lines of business (motor and fire) of the Nigerian insurance industry and two specific non-life insurance companies, indicates that the correlation coefficients vary and is generally weak. With the aid of the archimedean copula, the analysis makes use of the data pair exhibiting the highest correlation to draw particular attention to the importance of taking into account the extremal dependence structure when quantifying the risk capital, allocating risk and when estimating the net reinsurance premium under different reinsurance strategies.
\end{abstract}

Keywords: Tail dependent risks, Reinsurance treaties, Copulas, Economic capital, Stochastic simulation, Extreme value.

\section{Introduction}

When we consider more than one extremal event of interest, it is most often not a true reflection of reality to assume that the events are independent. Risk dependency can be seen between individual risks within each insurance portfolio and across lines of business (LoB) (Gildas, 2016). According to Li (2006), there are two broad sources of dependency between LoB - inherent dependency and other dependency. The inherent dependency is driven by factors such as inflation, interest rate, exchange rate, economic cycles and weather patterns. The other dependency arises as a result of the practices implemented by the insurer across its various LoB. Similar reserving methods and claims management procedures applied to different LoB are a few examples of such practices. Isaacs (2003) emphasizes the need for the insurer to properly account for dependence between risks stating that it can lead to inappropraite risk strategies as the insurer may be lulled to believe that he is less risky than he actually is.

\footnotetext{
${ }^{1}$ Author: Lecturer at the University of Uyo, Department of Insurance, Nigeria. Currently a doctoral student in financial mathematics at the Pan African University of Basic Sciences, Technology and Innovation Kenya.
} 
In this article, the tail dependence structure of large losses from the motor and fire insurance portfolios are evaluated. Using the copula model, the aggregation of risks which is an important factor in the determination of the capital requirement for both the insurer and the reinsurer, is addressed. The reason for choosing the motor and fire portfolios stem from the fact that in Nigeria, cases exist where truck tanks carrying flammable products, have collided with other cars leading to multiple motor accidents and fire explosions. A recent example occured in Lagos at the Odetola bridge where over 50 vehicles were burnt including lives lost due to a fuel tanker that had rammed into another car, skidded off the road, emptied its content on the ground and exploded (Ibrahim, 2018). Another event that could lead to motor and fire risks being dependent is the act of terrorism especially in crisis-ridden areas. There are situations were vehicles are shot at, some are battered and some are set ablaze. All these lead to car crashes, people injuries and burns. Thus, there is the need to evaluate and model the tail dependence structure of these losses in order to obtain more accurate inferences.

Using copulas, Frees and Valdez (1998) studied the dependence between liability insurance losses and allocated loss adjustment expenses (ALAE). Varying the insurer's retention at different values, they computed the reinsurance premium. Depuis and Jones (2006) used the same method and data to model the dependence of the joint upper tail of the distribution. Simulated hurricane losses in two U.S. states were investigated by Venter (2002). Belguise and Levi (2003) also made use of the copula technique to model the tail dependence between automobile and fire insurance losses. Other authors that have modelled the dependence between risk factors include Constantinescu et al.(2011), Denuit et al. (2005) and Tang and Valdez (2006).

To the best of the author's knowledge, there has been no study making use of African data that evaluates the extremal dependence structure of multiple lines of insurance claims and assesses its impact on reinsurance premium. This is the first research work done in this regard.

The structure of the paper is as follows - important copula concepts are presented along with the basic theory underlining the generalized Pareto distribution (GPD) in section 2. In section 3, the strength of the dependence for the three data pairs of losses (motor and fire) are analyzed. Illustrating using the motor and fire loss pair exhibiting the highest correlation, section 4 studies the impact of tail dependence on risk capital quantification and risk allocation. With the aid of stochastic simulation, section 5 focuses on estimating the net reinsurance premium under different reinsurance treaties in the presence of dependent risks and in each case, compares the results with those obtained when the risks are assumed to be independent. A summary of the analysis is presented in section 6 together with pathways to related future ideas.

\section{Extremes and Extremal Dependence}

This section reviews some basic concepts underlying the GPD and copulas. Tail dependence and some of the methods that can be used to estimate it are discussed. Then, the section is concluded with a description of the selected techniques for the application. 


\subsection{The Generalized Pareto distribution}

This is one of the more common techniques used in the EVT field for correctly choosing the data that lies in the extremes. Pickands (1975) developed the method.

Suppose $X_{1}, X_{2}, \ldots$ is a sequence of independent random variables with common distribution function $F$ and

$$
M_{n}=\max \left\{X_{1}, X_{2}, \ldots, X_{n}\right\}
$$

Then,

$$
\mathbb{P}\left[M_{n} \leq z\right] \leq z \rightarrow G(z) \quad \text { as } n \rightarrow \infty
$$

given that $X=\left\{X_{i}\right\}_{i=1}^{n}$ satisfies the generalized extreme value conditions.

$\mathrm{G}$ is a non-degenerate distribution function given as

$$
G(z)=\exp \left\{-\left(1+\xi\left(\frac{z-\mu}{\sigma}\right)\right)_{+}^{\frac{-1}{\xi}}\right\}
$$

for some $\mu, \sigma>0$ and $\xi$. For a large enough $u$ the distribution function of $X$ conditional on $X>u$ can be approximated by

$$
\mathbb{P}[X \leq y \mid X>u] \rightarrow H(y) \quad \text { as } u \rightarrow \infty
$$

where

$$
H(y)=1-\left(1+\xi \frac{y-u}{\sigma_{u}}\right)_{+}^{\frac{-1}{\xi}} \quad y>u
$$

$H(y)$ is the GPD with the modified scale parameter $\sigma_{u}=\sigma+\xi(u-\mu)$ corresponding to the excess of the threshold $u$. $z_{+}=\max (z, 0)$. For $X>u$ we can set $X=X-u$ and thus rewrite the equation as

$$
\mathbb{P}[X \leq y \mid X>u] \rightarrow H^{(u)}(y) \quad \text { as } u \rightarrow \infty
$$

where

$$
H^{(u)}(y)=1-\left(1+\xi \frac{y}{\sigma_{u}}\right)_{+}^{\frac{-1}{\xi}} \quad y>u
$$

For all cases, the GPD is given as

$$
\mathbb{P}(X<y \mid X>u)= \begin{cases}1-\left\{1+\xi\left(\frac{y-u}{\sigma_{u}}\right)\right\}_{+}^{\frac{-1}{\xi}} & \xi \neq 0 \\ 1-\exp \left\{-\frac{y-u}{\sigma_{u}}\right\}_{+} & \xi=0\end{cases}
$$

\subsection{The Concept of Tail Dependence}

Generally, dependence between two random variables $X$ and $Y$, imply that there is some kind of relationship between the variables. This implies that when we have information on $X$ we can say something about the $Y$ variable. Specifically, tail dependence focuses on the likelihood of one extreme 
event or a series of extreme events giving rise to another extreme event. Hence, according to Shaw et al. (2012), we are able to model the dependency between risks conditional on the underlying values of the risks themselves. Given that dependencies create riskier worlds, it is important to understand how the changes in an economy affect the dependency structure of risks and by extension, the economic capital of a firm. This will help in the evaluation of the amount of loss we may experience under very bad economic conditions versus under normal conditions.

\section{Tail Dependence Coefficient (TDC)}

This is a copula-based parameter developed by Ledford and Tawn (1996) which is defined as the probability that one risk variable $X_{i}$ takes an extreme value assuming that another risk variable $X_{j}$ also takes on an extreme value at the same probability level. Given the two dimensional random vector $\left(X_{i}, X_{j}\right)$ with marginal distribution functions $F_{i}$ and $F_{j}$, then the coefficient of upper tail dependence $\lambda_{u}$ and lower tail dependence $\lambda_{l}$ of $\left(X_{i}, X_{j}\right)$ respectively are:

$$
\begin{aligned}
& \lambda_{u}=\lim _{u \downarrow 1} \mathbb{P}\left(X_{i}>F_{i}^{-1}(u) \mid X_{j}>F_{j}^{-1}(u)\right) \\
& \lambda_{l}=\lim _{u \downarrow 0} \mathbb{P}\left(X_{i}<F_{i}^{-1}(u) \mid X_{j}<F_{j}^{-1}(u)\right)
\end{aligned}
$$

provided that the limit exists in both cases. $F_{i}^{-1}(u)$ and $F_{j}^{-1}(u)$ are the quantiles of risks $X_{i}$ and $X_{j}$ at level $u$. If $C$ is the copula of $\left(X_{i}, X_{j}\right)$ and $\bar{C}$ is the survival copula, we can represent $\lambda_{u}$ and $\lambda_{l}$ in terms of the copula function.

$$
\begin{gathered}
\lambda_{u}=\lim _{u \downarrow 1} \frac{\bar{C}(u, u)}{1-u}=\lim _{u \downarrow 1} \frac{1-2 u+C(u, u)}{1-u} \\
\lambda_{l}=\lim _{u \downarrow 1} \frac{C(u, u)}{u}
\end{gathered}
$$

Therefore, in general, we would have to use copulas with a higher tail dependence parameter if the variables are a lot more dependent than in a normal situation.

\subsubsection{Measures of Tail Dependence}

Traditionally, the linear (Pearson) correlation coefficient is used to describe the dependence between risks. Shaw et. al. (2012) argue that this measure falls short when we want to measure tail dependence because it does not represent all the possible relationships between risks. Moreover there is the need to understand the nature of the dependence structure. Other drawbacks of the Pearson correlation coefficient include the fact that it measures linear dependence and the assumption that must be satisfied by the random variables i.e. normally distributed. The Spearman rho and Kendall tau measures are better than the Pearson. More accurate measures have been developed to model tail dependence and compute the tail dependence coefficient.

\section{Copulas}


A copula $C$ is a joint distribution function of standard uniform random variables

$$
C\left(u_{1}, \ldots, u_{n}\right)=\mathbb{P}\left\{U 1 \leq u_{1}, \ldots, U_{d} \leq u_{n}\right\}
$$

where $U_{i}=U(0,1)$ for $i=1, \ldots, n$. Copulas allow one to characterize the dependence structure of a set of random variables separately from the marginal distributions.

Sklars theorem: This theorem forms the foundation for the use and application of copulas. Let $F$ be the joint distribution function of the random variables $X_{1}, \ldots, X_{n}$ with continuous marginal distribution functions $F_{1}\left(x_{1}\right), . ., F_{n}\left(x_{n}\right)$. Then, there exists a unique copula function $C$ given by

$$
C\left(u_{1}, \ldots, u_{n}\right)=F\left(x_{1}, \ldots, x_{n}\right)=F\left(F_{1}^{-1}\left(u_{1}\right), \ldots, F_{n}^{-1}\left(u_{n}\right)\right)
$$

The quantile function $F_{i}^{-1}$ is defined by $F_{i}^{-1}(u)=\inf \left\{x: F_{i}(x) \geq u\right\}$. Furthermore, the survival copula denoted as $\bar{C}$ is defined as,

$$
\mathbb{P}\left\{X_{1}>x_{1}, \ldots, X_{n}>x_{n}\right\}=\bar{C}\left(\mathbb{P}\left\{X_{1}>x_{1}\right\}, \ldots, \mathbb{P}\left\{X_{n}>x_{n}\right\}\right)
$$

In this study we will focus on the bivariate case. Thus for two random variables $X_{1}$ and $X_{2}$, the copula is a parameterization of the joint cumulative distribution function

$$
F\left(X_{1}, X_{2}\right)=\mathbb{P}\left(X_{1} \leq x_{1}, X_{2} \leq x_{2}\right)=C\left(F_{1}\left(x_{1}\right), F_{2}\left(x_{2}\right)\right)
$$

and the survival function can be expressed as

$$
\bar{C}\left(u_{1}, u_{2}\right)=u_{1}+u_{2}-1+C\left(1-u_{1}, 1-u_{2}\right)
$$

It should be noted that $\bar{C}$ is also a copula. It can also be used to model the dependence structure of two or more random variables. In this case it is termed the 'associated' copula. In general, though copulas are used to model the dependence structure of the whole distribution, it is also used to model tail dependence. The Archimedean and EV copulas are useful in this regard.

\section{Families of copulas}

\section{i. Elliptical copulas}

This family consists of the bivariate normal and bivariate $t$ copulas. The formula for the bivariate normal copula is

$$
C\left(u_{1}, u_{2}\right)=\Phi_{\rho}\left(\Phi^{-1}\left(u_{1}\right), \Phi^{-1}\left(u_{2}\right)\right)
$$

Where $\Phi_{\rho}$ is the standard normal distribution function for the bivariate case, having linear correlation 
$\rho . \Phi^{-1}$ is the inverse of the standard normal distribution function.

ii. The Archimedean copulas

This family of copulas is very useful when modeling tail dependence on one side, for instance, the upper tail dependence. The general representation is given by

$$
C\left(u_{1}, u_{2}\right)=\varphi^{-1}\left(\varphi\left(u_{1}\right)+\varphi\left(u_{2}\right)\right)
$$

The generator, $\varphi:(0,1] \rightarrow[0, \infty)$ is a strictly decreasing convex function with $\varphi(1)=0$. We assume that $\varphi^{-1}(t)=0$ for all $t \geq \lim _{u \downarrow 0} \varphi(u)$. Some well-known copulas in this family are the Clayton, Gumbel and Frank copulas.

iii. Extreme value copulas

For an independent and identically disributed (iid) sequence of random variables, the bivariate extreme value (BEV) copula come into play when we focus on the limit distribution of linearly normalized component wise maxima. It is represented by the formula:

$$
C\left(u_{1}, u_{2}\right)=\exp \left\{-V\left(\frac{-1}{\log u_{1}}, \frac{-1}{\log u_{2}}\right)\right\}
$$

where

$$
V(x, y)=\int_{0}^{1} \max \left\{\frac{w}{x}, \frac{1-w}{y}\right\} 2 d H(w)
$$

$H$ is a distribution function with support in $[0,1]$ and mean $\frac{1}{2}$.

The main limitation with the BEV copulas is that it cannot capture dependence structures that exhibit dependence at extreme values but independence in the limit (Depuis and Jones, 2006).

For an in-depth review on copulas see Frees and Valdez (1998) and Embrechts et.al. (2003)

\section{Archimedean copulas for insurance claims}

The most common Archimedean copulas are employed to compute the extremal dependence between two classes of insurance claims- auto and fire. That is, it is assumed that the portfolio is made up of these two classes of risks. The Archimedean copulas are used because we are particularly interested in understanding the dependence structure of two random variables in one region i.e. the joint upper tail. We will compare the results obtained with that from theindependence assumption to highlight the importance of incorporating tail dependence when modeling dependent insurance losses.

Clayton copula:

$$
C\left(u_{1}, u_{2}\right)=\left(u_{1}^{-\theta}+u_{2}^{-\theta}-1\right)^{\frac{-1}{\theta}}
$$

$\theta>0$ and the generator is $\varphi(t)=t^{-\theta}-1$

Gumbel copula:

$$
C\left(u_{1}, u_{2}\right)=\exp \left\{-\left(\left(-\ln u_{1}\right)^{\theta}+\left(-\ln u_{2}\right)^{\theta}\right)^{\frac{1}{\theta}}\right\}
$$


$\theta \geq 1$ and the generator is $\varphi(t)=(-\log t)^{\theta}$

Frank copula:

$$
C\left(u_{1}, u_{2}\right)=\frac{1}{\theta} \log \left(1+\frac{\left(e^{\theta u_{1}}-1\right)\left(e^{\theta u_{2}}-1\right)}{e^{\theta}-1}\right)
$$

$-\infty<\theta<\infty$ and the generator is $\varphi(t)=\log \frac{e^{\theta t}-1}{e^{\theta}-1}$

In this paper, only the Clayton and Gumbel copulas are used in the example.

\subsection{Aggregation of risks}

Two random variables $X_{i} \sim F_{i} \quad i=1,2$ are considered. $F_{i}=F$, the marginal distribution function, is known. In this case, it is the GPD. $X_{i}$ represents seperate classes of business that are correlated. If we set $X_{1}$ as $X$ and $X_{2}$ as $Y$ the the aggregate homogenous portfolio is given as

$$
S=X+Y
$$

The joint distribution of $(X, Y)$ is also specified. Thus we can uniquely determine the risk measures of the aggregate portfolio.

\subsubsection{Reinsurance premiums}

The XL reinsurance, stop loss (SL) reinsurance and a mix of both reinsurances are considered in this study. The XL reinsurance is represented as

$$
X L=\mathbb{E}\left\{\sum_{i=1}^{n}\left[\left(X_{i}+Y_{i}\right)-r\right]\right\}
$$

while stop loss is given as

$$
S L=\mathbb{E}\left\{\left[\sum_{i=1}^{n}\left(X_{i}+Y_{i}\right)-r\right]\right\}
$$

where $r>0$ is the retention level. $X_{i}$ and $Y_{i}$ represent motor and fire losses respectively.

\subsection{Risk measures}

To ensure that a firm continues operating under a worst-case scenario, the economic capital is the amount of capital that the firm must hold. A common way to determine this is by estimating the VaR at a remote probability. Other risk metrics that can be used to determine the economic capital include tail VaR (TVaR), excess TVaR (XTVaR) and expected policyholder (EPD).

$$
T V a R=\mathbb{E}[X \mid X \geq \mathrm{VaR}]=\frac{\int_{p}^{\infty} x f(x) d x}{1-p}
$$


Closely-related to TVaR is XTVaR given as TVaR minus the mean. In this case we assume that the mean is already financed by some other funding, thus we only need capital for the losses that exceed the mean.

Assuming the capital is set at VaR, EPD is then defined as the expected value of defaulted losses. That is,

$$
\mathrm{EPD}=(\mathrm{TVaR}-\mathrm{VaR})(1-p)
$$

EPD estimate is of importance to the policyholder because the policyholder is expected to bear the amount of loss in the situation where the insurer defaults on payment.

\section{Bivariate Analysis}

Data is obtained from Nigerian Insurance Commission (NAICOM 2013, 2014, 2015 and 2016) available at www.naicom.gov.ng. The large claims (3.5 million naira and above) are considered for years 2013 to 2016. First, the correlation between the individual losses for the industry-wide data and data from the Custodian and American International Insurance Company Limited (AIICO) are evaluated. Then, the pair with the highest correlation is used for illustrative purposes.

\subsection{Nonlinear correlations}

The dependence between motor and fire losses for the Nigerian insurance industry is positive but lower than that of AIICO, while that of the Custodian insurer is negative. The dependence structure varies from negative to positive correlations although the dependence is generally weak.

Table 1: Kendall tau and Spearman rho

\begin{tabular}{|r|r|r|}
\hline Motor and fire claims & kendall tau & Spearman rho \\
\hline Industry-wide data & 0.044 & 0.0662 \\
Custodian & -0.054 & -0.0838 \\
AIICO & 0.124 & 0.122 \\
\hline
\end{tabular}

\subsection{Illustrative example}

Amongst the selected insurers, the losses from AIICO exhibits the highest correlation (table 1). Thus it is used to illustrate the impact of dependency on some quantities that are of interest to the insurer. The four year data is made up of 132 losses for each risk.

The rank transform plot (figure 1) displays a distinct lack of data points in the upper-left (especially) and lower-right corners of the plot, although a general weak dependence can be observed. This indicates that the probability of a small fire claim accompanying a large motor claim and vice versa is low. In other words, the likelihood is higher for large losses or quantiles of motor to follow large losses or quantiles of fire and vice versa.

From the mep (figure 2), it can be seen that the same threshold of $4 \mathrm{e} 6$ is an appropraite threshold for 
Table 2: Descriptive statistics for AIICO's individual losses

\begin{tabular}{|r|r|r|}
\hline Industry data & motor & fire \\
\hline Min. & 3247500 & 42601 \\
1st Qu & 4050000 & 5050086 \\
Median & 4631500 & 7957982 \\
Mean & 5599483 & 18099085 \\
3rd Qu. & 5913000 & 15465777 \\
Max. & 51810400 & 345156235 \\
\hline AIICO & & \\
\hline Min. & 3247500 & 3509775 \\
1st Qu. & 4041112 & 5801860 \\
Median & 4569500 & 9674585 \\
Mean & 5206237 & 23048856 \\
3rd Qu. & 5636156 & 27801345 \\
Max. & 14073345 & 152991576 \\
\hline
\end{tabular}
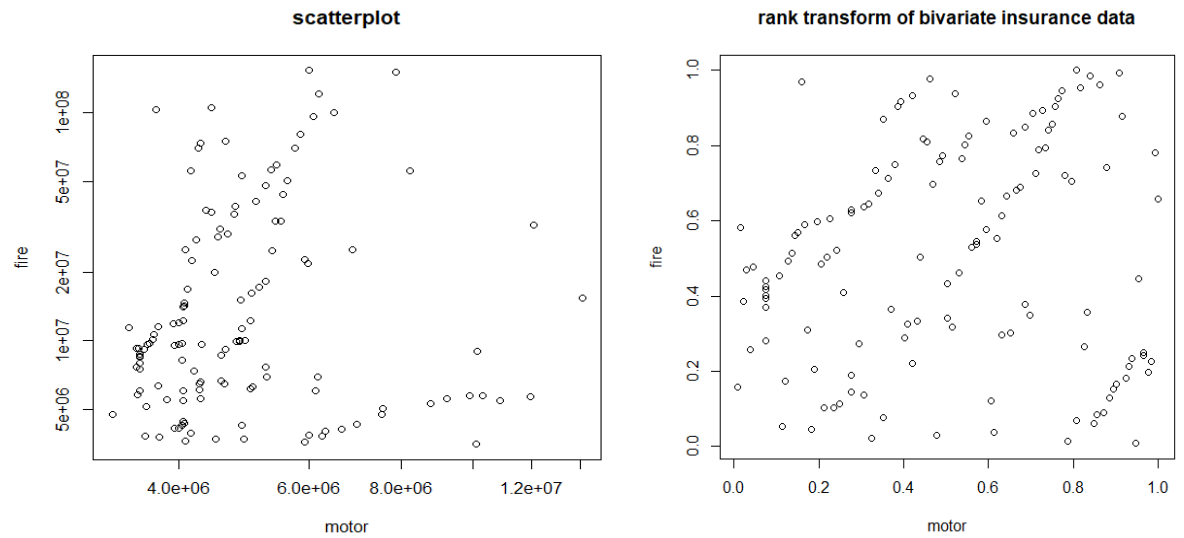

Figure 1: Scatterplot of AIICO log claims (left) and rank transformed data(right)

the individual losses. The GPD is fitted at this threshold and the model adequacy is confirmed by the goodness of fit (gof) plots (figure 2).
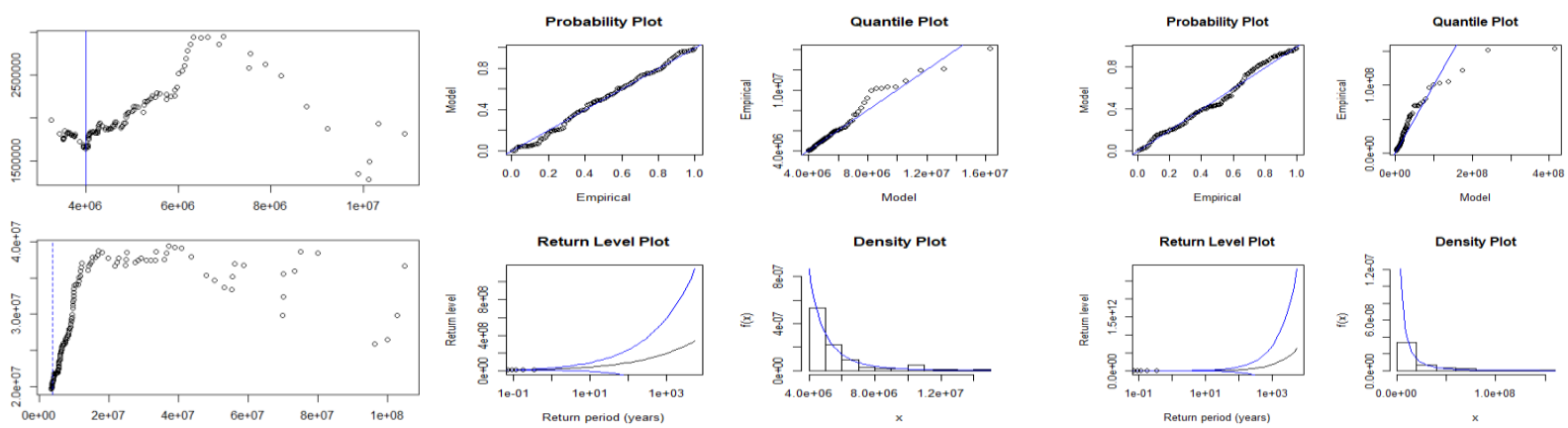

Figure 2: Left: Mean excess plot for motor (up) and fire (down). Center and right respectively: gof tests for motor and fire. 
Two bivariate distributions are created using the GPD parameters (table 3) and each of the selected copulas, then data is simulated from the distributions.

Table 3: GPD parameters

\begin{tabular}{|r|r|r|}
\hline & scale & shape \\
\hline motor & $1.158340 \mathrm{e} 6$ & 0.318 \\
fire & $8.307612 \mathrm{e} 6$ & 0.759 \\
\hline
\end{tabular}

\subsubsection{Estimating the parameters of the bivariate distribution}

Two methods are used to estimate the bivariate distribution - Maximum likelihood estimation (MLE) and Inference for margins (IFM). The estimated Clayton and Gumbel parameters with their respective standard errors are $0.285(0.004)$ and $1.14282(0.013)$.

Table 4: MLE and IFM estimation methods

\begin{tabular}{|c|rc|rc|c|c|}
\hline Parameters & \multicolumn{2}{|c|}{ motor } & \multicolumn{2}{c|}{ fire } & & \\
\hline & scale & shape & scale & shape & Clayton & Gumbel \\
\hline MLE & $1.158 \mathrm{e} 6$ & $0.3028(0.03)$ & $8.308 \mathrm{e} 6$ & $0.7434(0.01)$ & $0.2098(0.004)$ & \\
& $1.158 \mathrm{e} 6$ & $0.3103(0.03)$ & $8.308 \mathrm{e} 6$ & $0.7800(0.05)$ & & $1.162(0.013)$ \\
\hline IFM & $1.649 \mathrm{e} 6$ & $0.1444(0.03)$ & $2.776 \mathrm{e} 7$ & $0.3 .417(0.04)$ & 0.2856 & \\
& $1.670 \mathrm{e} 6$ & $0.1563(0.03)$ & $3.361 \mathrm{e} 7$ & $0.3172(0.04)$ & & 1.143 \\
\hline
\end{tabular}

For both the MLE and IFM rows in each case, the first line involves the Clayton copula while the second line is when the Gumbel is applied. The results indicate that the choice of the dependency structure plays an important part in the estimation of the bivariate distribution parameters. Though the values are similar under the MLE, there is a noticeable difference in the case of the IFM method.

\section{Quantifying Risk Capital and Allocating Risk}

The first six (of a thousand) entries of the simulated data used in each case (dependent and independent) to quantify the risk capital, allocate risk and compute the reinsurance premium is shown in tables 5 and 6. Aggregate losses in each line follows a GPD with the parameters given by the MLE estimated parameters. For the dependent case, the Clayton and Gumbel copulas are included in the model while the independence assumption is replicated by simply removing the copula dependency structure from the model.

Table 5: Simulated data for the independent case

\begin{tabular}{|r|r|r|r|}
\hline sim number & motor loss & fire loss & aggregate portfolio loss \\
\hline 1 & 5771942 & 22318941 & 28090883 \\
2 & 4286236 & 9309488 & 13595724 \\
3 & 5198114 & 36983903 & 42182017 \\
4 & 4147002 & 5399101 & 9546103 \\
5 & 4071796 & 5461946 & 9533742 \\
6 & 10088735 & 12223174 & 22311909 \\
\hline
\end{tabular}


Table 6: Simulated data for the dependent case (Clayton)

\begin{tabular}{|r|r|r|r|}
\hline sim number & motor loss & fire loss & aggregate portfolio loss \\
\hline 1 & 4414760 & 6749374 & 11164134 \\
2 & 6326208 & 17286991 & 23613199 \\
3 & 4663138 & 5707404 & 10370542 \\
4 & 7565872 & 47799368 & 55365241 \\
5 & 9294422 & 46886811 & 56181233 \\
6 & 4054413 & 7178607 & 11233020 \\
\hline
\end{tabular}
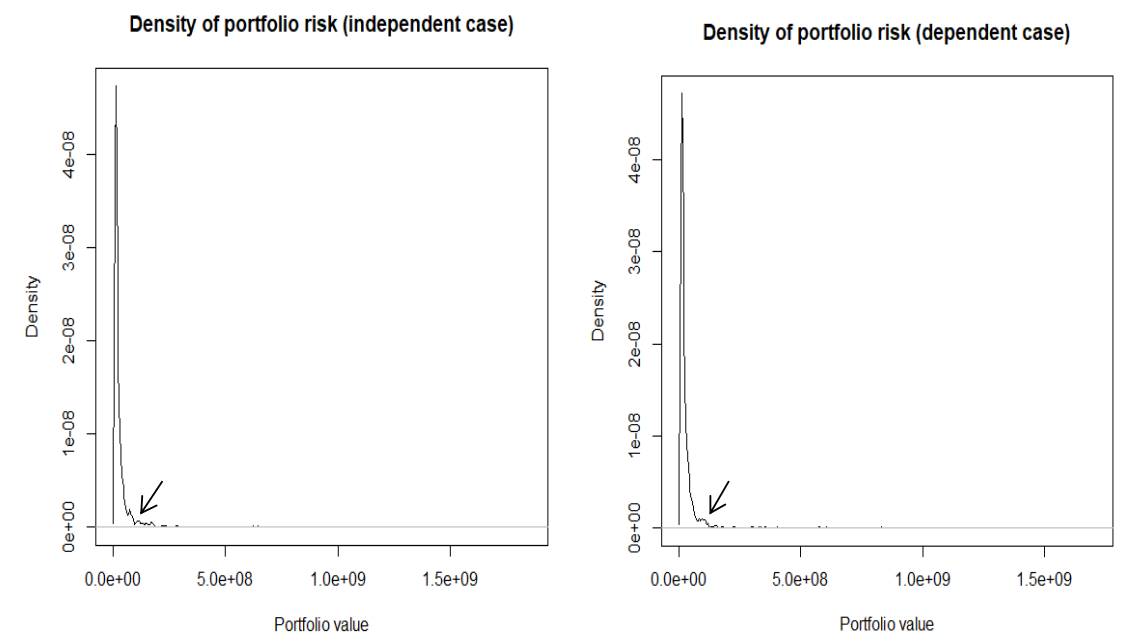

Figure 3: Density function of the aggregate losses: independent (left) and dependent (right) cases

The density plots for the both the independent and dependent (Clayton) cases look very much alike except at the tails. A noticeable difference can be seen at the points where the arrow is ponting at. The density function at this point is smoother with a slight bulge for the dependent case than that of the independent case which exhibits a slightly roughened edge. This is clearly depicted in the histograms below.
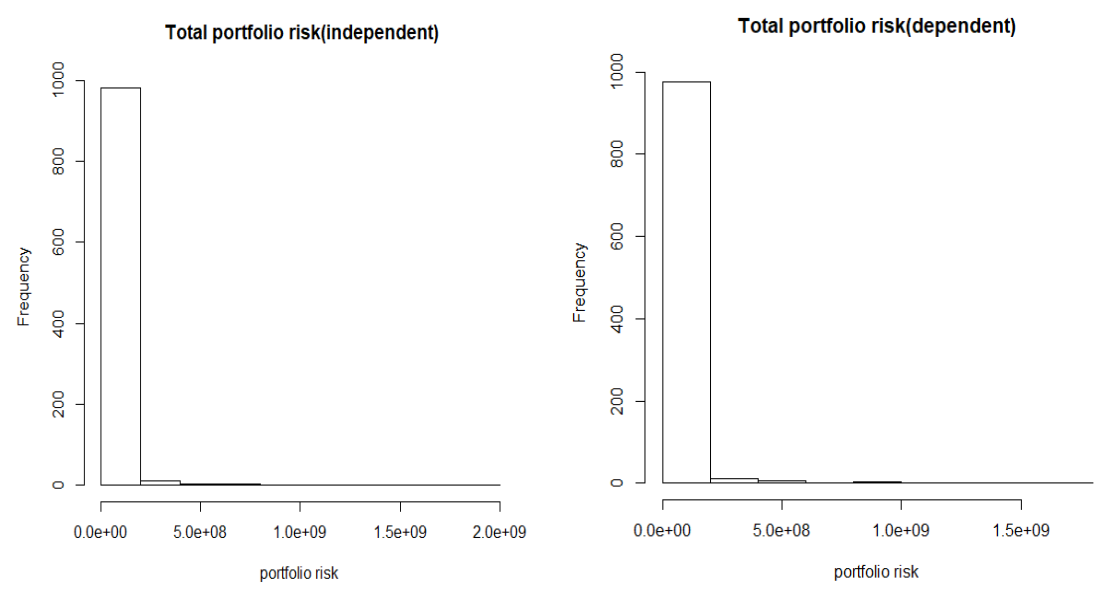

Figure 4: Histograms of the aggregate losses: independent (left) and dependent (right) cases 
Table 7: Risk capital for total risk

\begin{tabular}{|r|r|r|r|}
\hline Indep & $97 \%$ & $99 \%$ & $99.5 \%$ \\
\hline VaR & $156,678,722$ & $291,569,402$ & $575,659,669$ \\
TVaR & $377,052,095$ & $706,533,031$ & $959,021,998$ \\
XTVaR & $340,661,947$ & $670,142,883$ & $922,631,851$ \\
EPD & $6,611,201$ & $4,149,636$ & $1,916,812$ \\
\hline Dep (Clayton) & & & \\
\hline VaR & $153,178,320$ & $483,822,375$ & $605,701,183$ \\
TVaR & $448,168,109$ & $772,008,670$ & $984,129,772$ \\
XTVaR & $410,753,821$ & $734,594,381$ & $946,715,483$ \\
EPD & $8,849,694$ & $2,881,863$ & $1,892,143$ \\
\hline Dep (Gumbel) & & & \\
\hline VaR & $167,138,545$ & $349,112,469$ & $633,809,791$ \\
XVaR & $588,770,049$ & $1,272,882,384$ & $2,122,309,339$ \\
EPD & $545,484,629$ & $1,229,596,964$ & $2,079,023,919$ \\
& $12,648,945$ & $9,237,699$ & $7,442,498$ \\
\hline
\end{tabular}

Indep means independence, Dep means Dependence.

Table 8: Risk capital for individual risk

\begin{tabular}{|r|r|r|r|}
\hline & $97 \%$ & $99 \%$ & $99.5 \%$ \\
\hline Indep & & & \\
\hline VaR(motor) & $11,071,264$ & $16,566,065$ & $21,289,669$ \\
VaR(fire ) & $152,545,832$ & $286,563,060$ & $571,014,146$ \\
\hline Dep (Clayton) & & & \\
\hline VaR(motor) & $11,362,522$ & $15,698,533$ & $17,976,197$ \\
VaR(fire) & $145,111,384$ & $474,897,549$ & $595,986,491$ \\
\hline
\end{tabular}

The VaR, TVaR, XTVaR and EPD risk measures are evaluated (table 7). It is observed that when the tail dependence structure is quantified using the Gumbel copula, the risk measure estimates are all higher than in the case of the independence assumption. For the case of the Clayton copula, it is also generally higher for all other risk measures with the exception of the EPD risk is lower for for extreme quantiles when compared to the case of independence. In literature it has been shown that dependence among LoB increases the risk of the aggregate portfolio (see Cerchiara and Acri, 2016; Arachi and Belkacem, 2014). This is confirmed by the Clayton ( $V a R_{0.97}$ being an exception) and Gumbel copulas. Overall, less capital will be reserved when dependence is disregarded and this can lead to unanticipated solvency problems for the insurer. Another observation is that the VaR of the aggregate portfolio differs (is lower) from that of the sum of the individual losses and the difference widens at higher quantiles (table 8).

As an example, TVaR is allocated among LoB, given that it is a more useful risk measure than VaR (see Embrechts et al. 2014). The proportion of risk allocated to motor is computed using the simple formula

$$
\frac{T V a R_{\alpha}(\text { motor })}{T V a R_{\alpha}(\text { motor })+T V a R_{\alpha}(\text { fire })}
$$

Fire is calculated in a similar way or we simply subtract allocation for motor loss from one. 
Table 9: Risk allocation for motor loss

\begin{tabular}{|r|r|r|r|}
\hline & $97 \%$ & $99 \%$ & $99.5 \%$ \\
\hline Indep & 0.466 & 0.547 & 0.560 \\
Dep (Clayton) & 0.220 & 0.230 & 0.174 \\
Dep (Gumbel) & 0.062 & 0.035 & 0.023 \\
\hline
\end{tabular}

Allocating risk to different LoB is very useful to the insurer as it helps him to be able to set capacity controls, for example, premium targets and limits for each business unit. It also enables him to allocate capital that is in proportion to the specified risk measure. If independence between the loss variables is assumed when losses are actually correlated, the insurer will be led by this wrong assumption to allocate much more capital to motor claims instead of fire claims since the result indicates that the motor claims carry more weight when independence is assumed (table 9). In addition, an increasing trend can be noticed under the independence hypothesis while a decreasing trend is observed when tail dependence is accounted for. The choice of copula also affects the value of the estimate of the allocated risk.

\section{Premium Valuation}

In this section, 4 possible reinsurance treaties are considered under both the dependence and independence assumptions. They are as follows:

Option 1 No reinsurance treaty.

Option 2 Individual XL for each loss exceeding the specified layers.

Option 3 Mixed reinsurance treaty. Two cases are considered.

Case 1 - motor has proportional treaty covering $70 \%$ of the specified layers while fire has an aggregate XL treaty covering same layers.

Case 2 - fire has proportional treaty covering $70 \%$ of the specified layers while motor has an aggregate XL treaty covering same layers.

Option 4 Stop loss reinsurance covering the whole portfolio at the specified layers.

Only limited covers are considered for the three different layers in options 2 to 4 . The layers represent millions of naira. The stability of the result is quantified using standard error (SE) which can be seen in the braces.

Table 10: No reinsurance (option 1)

\begin{tabular}{|c|c|}
\hline & Option 1 \\
\hline Indep & $36,390,148(87,405,034)$ \\
Dep (Clayton) & $37,414,289(92,627,942)$ \\
Dep (Gumbel) & $43,285,420(187,224,002)$ \\
\hline
\end{tabular}

The impact of the choice of copula is made visible from the results displayed on table 11. In general, when the independent case is compared to dependent case, the difference between the reinsurance premium estimates in each case is not much. Additionally, if the dependence structure is specified 
Table 11: Net reinsurance premium (options 2-4)

\begin{tabular}{|c|c|c|c|c|}
\hline Layer & Option 2 & \multicolumn{2}{|c|}{ Option 3} & Option 4 \\
\hline Indep & & Case 1 & Case 2 & \\
\hline$(30,50]$ & $6,593,135(14,933,221)$ & $3,034,150(6,785,407)$ & $2,135,168(4,762,575)$ & $3,596,699(7,166,243)$ \\
\hline$(50,100]$ & $517,077(21,618,930)$ & $3,893,501(12,398,693)$ & $2,725,451(8,679,085)$ & $4,270,730(12,842,469)$ \\
\hline$(100,150]$ & $362,486(21,850,155)$ & $2,111,541(9,595,674)$ & $1,478,079(6,716,972)$ & $2,246,347(9,838,489)$ \\
\hline \multicolumn{5}{|c|}{$\overline{D e p}$ (Clayton) } \\
\hline$(30,50]$ & $255,698)$ & $2,780,838(6,423,849)$ & $1,951,207$ & $3,449,149(6,983,800)$ \\
\hline$(50,100]$ & $330,142(20,458,000)$ & $3,541,165(12,027,183)$ & $2,478,815(8,419,028)$ & $3,904,381(12,468,506)$ \\
\hline$(100,150]$ & $430,890(23,603,272)$ & $1,794,449(8,998,193)$ & $1,256,114(6,298,735)$ & $1,925,761(9,238,512)$ \\
\hline \multicolumn{5}{|c|}{ Dep (Gumbel) } \\
\hline$(30,50]$ & 98,429 & $860,976)$ & $2,170,264$ & $3,723,200(7,182,894)$ \\
\hline 1 & $6,980,472(2,2440,949)$ & $4,071,847(12,643,110)$ & $2,852,225(8,857,816)$ & $4,532,361(13,185,444)$ \\
\hline$(100,150]$ & $4,968,324(23,662,251)$ & $2,297,074(10,016,701)$ & $1,607,952(7,011,691)$ & $2,448,111(10,339,137)$ \\
\hline
\end{tabular}

using the Gumbel copula the reinsurance net premium is higher than in the clayton example. The SE also follows the same trend. For option 3, case 2 reports a lower net premium and SE than case 1. The reinsurance net premiums across the options indicate that option 3 case 2 gives the insurer the opportunity to pay the lowest premium along with the lowest SE while the highest premium to be paid occurs when option 2 is chosen. In a situation where the insurer decides not to take up reinsurance, the uncertainty associated with the mean aggregate claim shoots up (table 10).

A suprising find is observed under the mixed treaties and stop loss reinsurance (both independent and dependent assumptions). Here, the layer $(30,50]$ is generally lower than the $(50,100]$ layer with a much higher SE. This does not fall in line with the known fact which is, the higher the retention layer, the lower the reinsurance premium. This fact is only broadly confirmed under the individual XL reinsurance structure.

Eling and Toplek (2009) performed a similar analysis where they integrated several copula models and reinsurance contracts. Their simulation study revealed large differences in risk assessment under different copulas and reinsurance treaties. A similar situation is noted in this paper. Although the introduction of nonlinear dependencies led to a higher number of large losses in their analysis for all copulas when compared to the independent case, the situation differs a bit in this study given that both higher and lower losses are experienced when different copulas are employed. A point of note is that they focus on the expected gain of the insurer under the different reinsurance contracts while the focus here is on the expected losses above specified retention levels. Dhane and Goovearts (1996) showed that stop loss premium is higher when dependence is accounted for than under the independence assumption. In this study, the situation depends on the selected dependence structure.

Gildas (2016) studied loss ALAE claims and generally noted that the portfolio is less risky when independence is assumed. This find is confirmed true for a portfolio incorporating option 1 in this research (table 10). An interesting point raised in his study was that as the retention level is increased, the reinsurance premium for the XL treaty under different copula models tend to the estimates obtained under the independent assumption and that of the stop loss reinsurance deviates more from the independence results as deductible increases. In contrast, the result here shows that the reinsurance premiums under the copula models are much closer to the independent estimates when the lowest retention level is considered (table 11). 


\section{Conclusion}

A homogenous portfolio consisting of the aggregate bivariate losses from the Nigerian insurance sector was analyzed. The techniques employed were GPD and copulas. This paper showcased the impact of tail dependence on risk measures, risk allocation and reinsurance net premium. When losses are correlated, the value of the risk measure is increased and risk allocation is also affected. The choice of copulas also plays a major role as we can see reinsurance premium generally being lower than (in the case of the Clayton copula) and higher (in the case of the Gumbel copula) than the reinsurance premium obtained under the independent hypothesis.

A related problem would be to look at the inhomogeneous case where the $F_{i}$ 's are not necessarily the same. Another subject of investigation is dependence uncertainty which is a situation whereby the marginal distributions are fixed and known but the joint model is unspecified. 


\section{References}

Arachi, S. and Belkacem, L. (2014). Solvency Capital for Insurance Company: Modeling Dependence using Copula. Journal of Applied Economic Sciences. 9(4),30: 702-717.

Belguise, O. and Levi, C. (2003). Tempêtes: Étude des dépendances entre les branches Automobile et Incendie a` l'aide de la théorie des copules. ASTIN Colloquium, Berlin, Germany. www.astin 2003.de/03 call 03.shtml.

Cerchiara, R. R. and Acri, F. (2016). Aggregate Loss Distribution and Dependence: Composite Models, Copula Functions and Fast Fourier Transform for the Danish Fire Insurance Data. No. 201608. Working Papers. Universita della Calabria, Departimento di Economia, Statistica e Finanza. "Giovanni Anania" -DESF.

Constantinescu, C. Hashorva, E. and Ji, L. (2011) Archimedean copulas in Finite and Infinite Dimensionswith Application to Ruin Problems. Insurance Math. Econom., 49(3):487495. ISSN 0167-6687. doi: $10.1016 /$ j.insmatheco.

Depuis, D.J. and Jones, B. L. (2006). Multivariate Extreme Value Theory and its Usefulness in Understanding Risk. North American Actuarial Journal, Vol. 10, Issue 4.

Denuit, M., Dhaene, J., Goovaerts, M. and Kaas, R. (2005) Actuarial Theory for Dependent Risks: Measures, Orders and Models. Wiley.

Dhaene, J. and Goovaerts, M.J. (1996). Dependency of risks and stop-loss order. Astin Bulletin, 26(02):201212.

Embrechts, P., Filip L. and Mcneil A. (2003). Modeling Dependence with Copulas and Applications to Risk Management. In Handbook of Heavy Tailed Distributions in Finance, ed. S. T. Rachev, chap. 8, pp. 329-84. Amsterdam: Elsevier.

Embrechts, P. and Hofert M. (2013). Risk Measures and Dependence Modeling. In Handbook of Insurance, Springer 135-165.

Eling, M. and Toplek, D. (2009). Risk and Return of Reinsurance Contracts under copula Models. The European Journal of Finance, Vol. 15, 751-775. Taylor and Francis Group.

Frees, E. W. and Valdez, E. A. (1998). Understanding Relationships using Copulas. North American Actuarial Journal 2(1): 1-25.

Li, J. (2006). Modeling Dependency between different Lines of Business with Copulas. Research Paper 146.

Gildas, R. (2016). Mixed Erlang Reinsurance Risk: Aggregation, Capital allocation and Default risk. The European Actuarial Journal 6 (1):149-175.

Gildas, R. (2016). Modeling Dependent Insurance Risks. Thesis, University of Lausanne.

Ibrahim, A. A. (2018). Lagos Tanker Explosion: Smell of Burnt Human Flesh filled the Air - Reporter's Account. Retrieved on the July 8th from Punchng.com

Isaacs, D. (2003). Capital Adequacy and Dependence. Paper presented to the Institute of Actuaries of Austrailia XIV General Insurance Seminar.

Pickands, J. (1975). Statistical Inference using Extreme Order Statistics. Annals of Statistics 3(1): 
$119-31$.

Shaw, R. A., Smith, A. D. and Spivak, G. S. (2012). Measurement and Modelling of Dependencies in Economic Capital. British Actuarial Journal, 16(3), 601-699.

Tang, A. and Valdez, E. A. (2006). Economic Capital and the Aggregation of Risks using Copulas. In Proceedings of the 28th International Congress of Actuaries, Paris France, 2006.

Venter, G. G.( 2002). Tails of Copulas. Proceedings of the Casualty Actuarial Society 89: 68-113. 